\title{
On Choosing Ferrocene as an Internal Reference Redox Scale for Voltammetric Measurements: A Cautionary Tale
}

\section{Angel AJ Torriero*}

School of Life and Environmental Sciences, Deakin University, Australia

*Corresponding author: Angel A J Torriero, School of Life and Environmental Sciences, Deakin University, Burwood, Victoria 3125, Australia, Tel: +61392446897;

Email: angel.torriero@deakin.edu.au

\section{Mini Review \\ Volume 3 Issue 4}

Received Date: November 25, 2019

Published Date: December 18, 2019

DOI: $10.23880 /$ macij- 16000151

\section{Abbreviations: CV: Cyclic Voltammetry.}

\section{Introduction}

Internal reference redox scales or internal reference redox systems, IRRS, are reversible or nearly reversible redox systems used to provide a known and stable reference point in non-aqueous solvents in conditions where reliable reference electrodes are difficult to be established and/or stabilized [1,2]. In general, the IRRS is used in conjunction with quasi-reference electrodes [3-8]. They have a long story, which began with the concept of $\mathrm{Rb} \mid \mathrm{Rb}^{+}$or $\mathrm{Rb}(\mathrm{Hg}) \mid \mathrm{Rb}^{+}$, followed by the use of organometallic redox couples [9]. In order to limit the number of redox systems used as IRRS and then made easier the comparison, IUPAC recommended in 1983 that the systems ferrocene|ferrocenium, $\mathrm{Fc}^{0 /+}$, and bis(biphenyl)chromium(0)|bis(biphenyl)chromium(I), $\mathrm{BCr}^{0 /+}$, need to be used as internal reference redox systems in non-aqueous media. These two complexes were selected arbitrarily from several published redox systems.

An effective IRRS must possess a number of properties. Many of which were suggested by Gritzner and Kuta in their IUPAC recommendation [9], and most of which can be deduced by common sense. We recently suggested the following to be essential properties of an IRRS [10]:

- The $\mathrm{OX}_{\text {IRRS }} / \mathrm{RED}_{\mathrm{IRRS}}$ redox couple $\left(\mathrm{OX}_{\mathrm{IRRS}}+\mathrm{e}^{-} \leftrightarrows \mathrm{RED}_{\mathrm{IRRS}}\right.$; being $O X_{\text {IRRS }}$ and $\mathrm{RED}_{\text {IRRS }}$ the oxidised and reduced form of the IRRS, respectively) must be reversible or nearly reversible under the operative measurement conditions. It is advantageous for the IRRS electron transfer to be a simple one-electron outer-sphere electron transfer.

- It is very important that the IRRS redox moieties do not specifically interact with the working electrode (e.g., they do not adsorb on the working electrode).

- The mid-point potential for the IRRS should not overlap the active potential zone of the analyte (ANS) under study.

- The initially present IRRS redox component, $\mathrm{OX}_{\mathrm{IRRS}}$ or

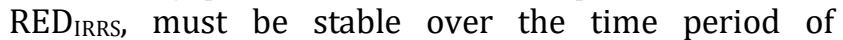
experimentation; it should not react (or interact) with the solvent, supporting electrolyte or ANS.

- The IRRS redox moiety, $\mathrm{OX}_{\text {IRRS }}$ or $\mathrm{RED}_{\text {IRRS, which is not }}$ initially present, must be stable on the time scale required to execute the slowest cyclic voltammetry (CV).

- The molecule or salt that is the source of the selected IRRS component should be pure, easily synthesized, or otherwise, readily available and should have a long shelf life.

There are also some constraints and requirements for the ANS [10]:

- The ANS is initially present as either an oxidizable moiety $\left(R E D_{A N S}\right)$ or reducible moiety $\left(O X_{A N S}\right)$. It is worth noting that $\mathrm{RED}_{\mathrm{ANS}}$ and $\mathrm{OX}_{\mathrm{ANS}}$ species may or may not be redox partners, and the oxidation of $R_{E D} D_{A N S}$ and reduction of $\mathrm{OX}_{\mathrm{ANS}}$ may be complicated.

- The initially present ANS moiety should be stable and should not react with the IRRS.

It is necessary to notice that IUPAC recommendation has been proposed for organic solvents and their mixtures. By extrapolation from those systems, the $\mathrm{Fc}^{0 /+}$ redox couple is also being used as an IRRS in ionic liquids, ILs $[2,11-13,3]$. 


\section{Medicinal \& Analytical Chemistry International Journal}

Nowadays, it is well known that Fc is not an 'ideal' redox process as mentioned in the IUPAC recommendation, as its formal potential is highly dependent on the solvation effects of the organic solvent and supporting electrolyte used, which includes the electrostatic interaction of solvent/electrolyte with the iron centre and the cyclopentadienyl ring $[14,15]$.

Decamethylferrocene (DmFc or $\left.\left[\mathrm{Fe}^{\mathrm{Il}}\left(\eta^{5}-\mathrm{C}_{5}\left(\mathrm{CH}_{3}\right)_{5}\right)_{2}\right]\right)$ shows at least an order of magnitude weaker solventsolute interaction compared to ferrocene, as the methylsubstituent groups in the cyclopentadienyl rings prevent both specific and non-specific interactions by hindering the access of organic solvent and supporting electrolyte molecules to the metal centre and to the cyclopentadienyl ring [14,16-19]. This observation was also confirmed after analysis of the X-ray diffraction patterns of decamethylferrocene, which indicate that the inter-ring methyl groups of $\mathrm{DmFc}$ are within Van der Waals distances [20]. Consequently, DmFc is better suited than $\mathrm{Fc}$ as an IRRS in organic solvents.

Decamethylferrocene is oxidized in organic solvents in a reversible, one-electron transfer, process to decamethylferrocenium $\left(\mathrm{DmFc}^{+}\right.$or $\left.\left[\mathrm{Fe}^{\mathrm{III}}\left(\eta^{5}-\mathrm{C}_{5}\left(\mathrm{CH}_{3}\right)_{5}\right)_{2}\right]^{+}\right)$ and reduced back to $\mathrm{DmFc}$ according to Equation 1. The midpoint potential $\left(E_{m}\right)$, calculated from the average of the oxidation $\left(\mathrm{E}_{\mathrm{p}} \mathrm{ox}\right)$ and reduction $\left(\mathrm{E}_{\mathrm{p}}\right.$ red $)$ peak potentials $\left[\left(\mathrm{E}_{\mathrm{p}}{ }^{\mathrm{ox}}+\mathrm{E}_{\mathrm{p}}{ }^{\mathrm{red}}\right) / 2\right]$,of this redox couple is more negative than that of $\mathrm{Fc}$ as a consequence of the electron-donating effect of the methyl groups (inductive effect), which by pushing electron density towards the metal ion facilitates the electron removal by the electrode. As given in Table 1, the difference in the $E_{m}$ values between $\mathrm{Fc}^{0 /+}$ and $\mathrm{DmFc}^{0 /+}$ varies from a low of $0.413 \pm 0.005 \mathrm{~V}$ in tetrahydrofuran/0.1M $\left[\mathrm{Bu}_{4} \mathrm{~N}\right]\left[\mathrm{BF}_{4}\right]$ to a high of $0.614 \pm$ $0.005 \mathrm{~V}$ in dichloromethane/0.1M [Bu $\left.\mathrm{Bu}_{4} \mathrm{~N}\right][\mathrm{TFAB}]$ ([TFAB] $\left.=\left[\mathrm{B}\left(\mathrm{C}_{6} \mathrm{~F}_{5}\right)_{4}\right]^{-}\right)$, which represents a difference of $0.201 \mathrm{~V}$. Furthermore, a variation of about $0.152 \mathrm{~V}$ can be observed by changing the organic solvent from tetrahydrofuran to 2,2,2-trifluoroethanol and keeping constant the supporting electrolyte nature and concentration $\left(0.1 \mathrm{M} \quad\left[\mathrm{Bu}_{4} \mathrm{~N}\right]\left[\mathrm{ClO}_{4}\right]\right)$. To minimize uncertainties in the comparison of mid-point potentials in Table 1, all potentials quoted are relative to the $\mathrm{DmFc}^{0 /+}$ potential scale. Consequently, since the values presented in this table represent the difference in $E_{m}$, the variation in the absolute values of $E_{m}$ of the $\mathrm{Fc}^{0 /+}$ and $\mathrm{DmFc}^{0 /+}$ IRRS may probably be larger than those indicated here.

$$
\left[\mathrm{Fe}^{\mathrm{III}}\left(\eta^{5}-\mathrm{C}_{5}\left(\mathrm{CH}_{3}\right)_{5}\right)_{2}\right] \leftrightarrows\left[\mathrm{Fe}^{\mathrm{III}}\left(\eta^{5}-\mathrm{C}_{5}\left(\mathrm{CH}_{3}\right)_{5}\right)_{2}\right]^{+}+\mathrm{e}^{-}
$$

\begin{tabular}{|c|c|c|c|}
\hline Solvent & Electrolyte & $\mathrm{Fc}^{0 /+}$ vs. $\mathrm{DmFc}^{0 /+}(\mathrm{V})$ & Ref \\
\hline \multirow{2}{*}{ Diethyl ether } & $0.1 \mathrm{M}\left[\mathrm{Bu}_{4} \mathrm{~N}\right]\left[\mathrm{BArF}_{24}\right]^{a}$ & $0.550 \pm 0.005$ & [15] \\
\hline & $0.1 \mathrm{M} \mathrm{Na}\left[\mathrm{BArF}_{24}\right]^{a}$ & $0.583 \pm 0.005$ & [15] \\
\hline \multirow{2}{*}{ Anisole } & $0.1 \mathrm{M}\left[\mathrm{Bu}_{4} \mathrm{~N}\right]\left[\mathrm{PF}_{6}\right]$ & $0.518 \pm 0.005$ & [15] \\
\hline & $0.1 \mathrm{M}\left[\mathrm{Bu}_{4} \mathrm{~N}\right]\left[^{\mathrm{TFAB}}\right]^{b}$ & $0.607 \pm 0.005$ & {$[15]$} \\
\hline Methanol & $0.1 \mathrm{M}\left[\mathrm{Bu}_{4} \mathrm{~N}\right]\left[\mathrm{ClO}_{4}\right]$ & $0.497 \pm 0.002$ & {$[14]$} \\
\hline Ethanol & $0.1 \mathrm{M}\left[\mathrm{Bu}_{4} \mathrm{~N}\right]\left[\mathrm{ClO}_{4}\right]$ & $0.473 \pm 0.005$ & [14] \\
\hline 2,2,2-trifluoroethanol & $0.1 \mathrm{M}\left[\mathrm{Bu}_{4} \mathrm{~N}\right]\left[\mathrm{ClO}_{4}\right]$ & $0.575 \pm 0.004$ & [14] \\
\hline 2-propanol & $0.1 \mathrm{M}\left[\mathrm{Bu}_{4} \mathrm{~N}\right]\left[\mathrm{CF}_{3} \mathrm{SO}_{3}\right]$ & $0.455 \pm 0.003$ & [14] \\
\hline \multirow{9}{*}{ Tetrahydrofuran } & $0.1 \mathrm{M}\left[\mathrm{Bu}_{4} \mathrm{~N}\right]\left[\mathrm{BF}_{4}\right]$ & $0.413 \pm 0.005$ & [15] \\
\hline & $0.1 \mathrm{M}\left[\mathrm{Bu}_{4} \mathrm{~N}\right]\left[\mathrm{CF}_{3} \mathrm{SO}_{3}\right]$ & $0.438 \pm 0.005$ & [15] \\
\hline & \multirow{2}{*}{$0.1 \mathrm{M}\left[\mathrm{Bu}_{4} \mathrm{~N}\right]\left[\mathrm{ClO}_{4}\right]$} & $0.423 \pm 0.005$ & [15] \\
\hline & & $0.427 \pm 0.002$ & {$[14]$} \\
\hline & $0.1 \mathrm{M}\left[\mathrm{Bu}_{4} \mathrm{~N}\right]\left[\mathrm{PF}_{6}\right]$ & $0.446 \pm 0.005$ & [15] \\
\hline & $0.1 \mathrm{M}\left[\mathrm{Bu}_{4} \mathrm{~N}\right]\left[\mathrm{BPh}_{4}\right]$ & $0.485 \pm 0.005$ & [15] \\
\hline & $0.1 \mathrm{M} \mathrm{Na}\left[\mathrm{BArF}_{24}\right]^{a}$ & $0.502 \pm 0.005$ & [15] \\
\hline & $0.1 \mathrm{M}\left[\mathrm{Bu}_{4} \mathrm{~N}\right]\left[\mathrm{TFAB}^{b}\right.$ & $0.484 \pm 0.005$ & [15] \\
\hline & $0.1 \mathrm{M}\left[\mathrm{Bu}_{4} \mathrm{~N}\right]\left[\mathrm{BArF}_{24}\right]^{a}$ & $0.521 \pm 0.005$ & [15] \\
\hline \multirow[t]{7}{*}{ Pyridine } & $0.1 \mathrm{M}\left[\mathrm{Bu}_{4} \mathrm{~N}\right]\left[\mathrm{ClO}_{4}\right]$ & $0.517 \pm 0.004$ & [14] \\
\hline & $0.1 \mathrm{M}\left[\mathrm{Bu}_{4} \mathrm{~N}\right] \mathrm{Cl}$ & $0.534 \pm 0.005$ & [15] \\
\hline & $0.1 \mathrm{M}\left[\mathrm{Bu}_{4} \mathrm{~N}\right]\left[\mathrm{ClO}_{4}\right]$ & $0.532 \pm 0.002$ & [14] \\
\hline & $0.1 \mathrm{M}\left[\mathrm{Bu}_{4} \mathrm{~N}\right]\left[\mathrm{PF}_{6}\right]$ & $0.548 \pm 0.003$ & [21] \\
\hline & $0.1 \mathrm{M}\left[\mathrm{Et}_{4} \mathrm{~N}\right]\left[\mathrm{BF}_{4}\right]$ & $0.541 \pm 0.003$ & [22] \\
\hline & $0.1 \mathrm{M}\left[\mathrm{Bu}_{4} \mathrm{~N}\right][\mathrm{TFAB}]^{b}$ & $0.614 \pm 0.005$ & [15] \\
\hline & $0.1 \mathrm{M}\left[\mathrm{C}_{4} \mathrm{mPyr}\right][\mathrm{FAP}]$ & $0.589 \pm 0.003$ & [22] \\
\hline
\end{tabular}




\section{Medicinal \& Analytical Chemistry International Journal}

\begin{tabular}{|c|c|c|c|}
\hline \multirow{10}{*}{ Dichloromethane } & $0.1 \mathrm{M}\left[\mathrm{C}_{2} \mathrm{mim}\right][\mathrm{FAP}]$ & $0.590 \pm 0.003$ & [22] \\
\hline & $0.1 \mathrm{M}\left[\mathrm{C}_{2} \mathrm{mim}\right]\left[\mathrm{B}(\mathrm{CN})_{4}\right]$ & $0.588 \pm 0.003$ & [22] \\
\hline & $0.1 \mathrm{M}\left[\mathrm{C}_{4} \mathrm{mim}\right]\left[\mathrm{N}(\mathrm{Tf})_{2}\right]$ & $0.570 \pm 0.003$ & [22] \\
\hline & $0.1 \mathrm{M}\left[\mathrm{C}_{4} \mathrm{mPyr}\right]\left[\mathrm{N}(\mathrm{Tf})_{2}\right]$ & $0.568 \pm 0.003$ & [22] \\
\hline & $0.1 \mathrm{M}\left[\mathrm{C}_{2} \mathrm{mim}\right][\mathrm{FSI}]$ & $0.569 \pm 0.003$ & [22] \\
\hline & $0.1 \mathrm{M}\left[\mathrm{C}_{3} \mathrm{mim}\right][\mathrm{FSI}]$ & $0.568 \pm 0.003$ & [22] \\
\hline & $0.1 \mathrm{M}\left[\mathrm{C}_{4} \mathrm{mPyr}\right]\left[\mathrm{N}(\mathrm{CN})_{2}\right]$ & $0.564 \pm 0.003$ & [22] \\
\hline & $0.1 \mathrm{M}\left[\mathrm{C}_{4} \mathrm{mim}\right]\left[\mathrm{PF}_{6}\right]$ & $0.556 \pm 0.003$ & [22] \\
\hline & $0.1 \mathrm{M}\left[\mathrm{C}_{4} \mathrm{mim}\right]\left[\mathrm{BF}_{4}\right]$ & $0.557 \pm 0.003$ & [22] \\
\hline & $0.1 \mathrm{M}\left[\mathrm{C}_{4} \mathrm{mim}\right]\left[\mathrm{CF}_{3} \mathrm{SO}_{3}\right]$ & $0.556 \pm 0.003$ & [22] \\
\hline 1,2-dichloroethane & $0.1 \mathrm{M}\left[\mathrm{Bu}_{4} \mathrm{~N}\right]\left[\mathrm{ClO}_{4}\right]$ & $0.532 \pm 0.001$ & [14] \\
\hline 1,2-dibromoethane & $0.1 \mathrm{M}\left[\mathrm{Bu}_{4} \mathrm{~N}\right]\left[\mathrm{ClO}_{4}\right]$ & $0.475 \pm 0.007$ & [14] \\
\hline \multirow{4}{*}{ Benzonitrile } & $0.1 \mathrm{M}\left[\mathrm{Bu}_{4} \mathrm{~N}\right] \mathrm{Cl}$ & $0.524 \pm 0.005$ & [15] \\
\hline & $0.1 \mathrm{M}\left[\mathrm{Bu}_{4} \mathrm{~N}\right]\left[\mathrm{ClO}_{4}\right]$ & $0.523 \pm 0.001$ & [14] \\
\hline & $0.1 \mathrm{M}\left[\mathrm{Bu}_{4} \mathrm{~N}\right]\left[\mathrm{PF}_{6}\right]$ & $0.530 \pm 0.005$ & [15] \\
\hline & $0.1 \mathrm{M}\left[\mathrm{Bu}_{4} \mathrm{~N}\right]\left[\mathrm{TFAB}^{b}\right.$ & $0.543 \pm 0.005$ & [15] \\
\hline Bromobenzene & $0.1 \mathrm{M}\left[\mathrm{Bu}_{4} \mathrm{~N}\right]\left[\mathrm{ClO}_{4}\right]$ & $0.489 \pm 0.005$ & [14] \\
\hline Chlorobenzene & $0.1 \mathrm{M}\left[\mathrm{Bu}_{4} \mathrm{~N}\right]\left[\mathrm{ClO}_{4}\right]$ & $0.497 \pm 0.001$ & [14] \\
\hline 1,2-dichlorobenzene & $0.1 \mathrm{M}\left[\mathrm{Bu}_{4} \mathrm{~N}\right]\left[\mathrm{ClO}_{4}\right]$ & $0.535 \pm 0.001$ & [14] \\
\hline Benzyl alcohol & $0.1 \mathrm{M}\left[\mathrm{Bu}_{4} \mathrm{~N}\right]\left[\mathrm{ClO}_{4}\right]$ & $0.508 \pm 0.003$ & [14] \\
\hline Nitrobenzene & $0.1 \mathrm{M}\left[\mathrm{Bu}_{4} \mathrm{~N}\right]\left[\mathrm{ClO}_{4}\right]$ & $0.514 \pm 0.002$ & [14] \\
\hline Aniline & $0.1 \mathrm{M}\left[\mathrm{Bu}_{4} \mathrm{~N}\right]\left[\mathrm{ClO}_{4}\right]$ & $0.527 \pm 0.004$ & [14] \\
\hline Toluene & {$\left[\mathrm{Bu}_{4} \mathrm{~N}\right]\left[\mathrm{BF}_{4}\right]^{c}$} & $0.430 \pm 0.005$ & [15] \\
\hline \multirow{4}{*}{ Acetone } & $0.1 \mathrm{M}\left[\mathrm{Bu}_{4} \mathrm{~N}\right] \mathrm{Cl}$ & $0.451 \pm 0.005$ & [15] \\
\hline & $0.1 \mathrm{M}\left[\mathrm{Bu}_{4} \mathrm{~N}\right]\left[\mathrm{ClO}_{4}\right]$ & $0.479 \pm 0.004$ & [14] \\
\hline & $0.1 \mathrm{M}\left[\mathrm{Bu}_{4} \mathrm{~N}\right]\left[\mathrm{PF}_{6}\right]$ & $0.487 \pm 0.005$ & [15] \\
\hline & $0.1 \mathrm{M}\left[\mathrm{Bu}_{4} \mathrm{~N}\right][\mathrm{TFAB}]^{b}$ & $0.504 \pm 0.005$ & [15] \\
\hline Propylene carbonate & $0.1 \mathrm{M}\left[\mathrm{Bu}_{4} \mathrm{~N}\right]\left[\mathrm{ClO}_{4}\right]$ & $0.495 \pm 0.002$ & [14] \\
\hline \multirow{4}{*}{ Acetonitrile } & $0.1 \mathrm{M}\left[\mathrm{Bu}_{4} \mathrm{~N}\right] \mathrm{Cl}$ & $0.501 \pm 0.005$ & [15] \\
\hline & $0.1 \mathrm{M}\left[\mathrm{Bu}_{4} \mathrm{~N}\right]\left[\mathrm{ClO}_{4}\right]$ & $0.505 \pm 0.002$ & [14] \\
\hline & $0.1 \mathrm{M}\left[\mathrm{Bu}_{4} \mathrm{~N}\right]\left[\mathrm{PF}_{6}\right]$ & $0.509 \pm 0.003$ & [21] \\
\hline & $0.1 \mathrm{M}\left[\mathrm{Bu}_{4} \mathrm{~N}\right][\mathrm{TFAB}]^{b}$ & $0.517 \pm 0.005$ & [15] \\
\hline \multirow{4}{*}{ Nitromethane } & $0.1 \mathrm{M}\left[\mathrm{Bu}_{4} \mathrm{~N}\right] \mathrm{Cl}$ & $0.505 \pm 0.005$ & [15] \\
\hline & $0.1 \mathrm{M}\left[\mathrm{Bu}_{4} \mathrm{~N}\right]\left[\mathrm{ClO}_{4}\right]$ & $0.516 \pm 0.004$ & [14] \\
\hline & $0.1 \mathrm{M}\left[\mathrm{Bu}_{4} \mathrm{~N}\right]\left[\mathrm{PF}_{6}\right]$ & $0.510 \pm 0.005$ & [15] \\
\hline & $0.1 \mathrm{M}\left[\mathrm{Bu}_{4} \mathrm{~N}\right]\left[\mathrm{TFAB}^{b}\right.$ & $0.516 \pm 0.005$ & [15] \\
\hline Formamide & $0.1 \mathrm{M}\left[\mathrm{Bu}_{4} \mathrm{~N}\right]\left[\mathrm{ClO}_{4}\right]$ & $0.510 \pm 0.003$ & [14] \\
\hline N-methylformamide & $0.1 \mathrm{M}\left[\mathrm{Bu}_{4} \mathrm{~N}\right]\left[\mathrm{ClO}_{4}\right]$ & $0.510 \pm 0.002$ & [14] \\
\hline \multirow{4}{*}{$\mathrm{N}, \mathrm{N}$-dimethylformamide } & $0.1 \mathrm{M}\left[\mathrm{Bu}_{4} \mathrm{~N}\right] \mathrm{Cl}$ & $0.475 \pm 0.005$ & [15] \\
\hline & $0.1 \mathrm{M}\left[\mathrm{Bu}_{4} \mathrm{~N}\right]\left[\mathrm{ClO}_{4}\right]$ & $0.458 \pm 0.003$ & [14] \\
\hline & $0.1 \mathrm{M}\left[\mathrm{Bu}_{4} \mathrm{~N}\right]\left[\mathrm{PF}_{6}\right]$ & $0.478 \pm 0.005$ & [15] \\
\hline & $0.1 \mathrm{M}\left[\mathrm{Bu}_{4} \mathrm{~N}\right][\mathrm{TFAB}]^{b}$ & $0.493 \pm 0.005$ & [15] \\
\hline $\mathrm{N}, \mathrm{N}$-dimethylacetamide & $0.1 \mathrm{M}\left[\mathrm{Bu}_{4} \mathrm{~N}\right]\left[\mathrm{ClO}_{4}\right]$ & $0.455 \pm 0.008$ & [14] \\
\hline \multirow{3}{*}{ Dimethyl sulfoxide } & $0.1 \mathrm{M}\left[\mathrm{Bu}_{4} \mathrm{~N}\right]\left[\mathrm{PF}_{6}\right]$ & $0.486 \pm 0.005$ & [15] \\
\hline & $0.1 \mathrm{M}\left[\mathrm{Bu}_{4} \mathrm{~N}\right]\left[\mathrm{TFAB}^{b}\right.$ & $0.493 \pm 0.005$ & [15] \\
\hline & $0.1 \mathrm{M}\left[\mathrm{Bu}_{4} \mathrm{~N}\right]\left[\mathrm{ClO}_{4}\right]$ & $0.468 \pm 0.001$ & [14] \\
\hline Chloroform & $0.1 \mathrm{M}\left[\mathrm{Bu}_{4} \mathrm{~N}\right]\left[\mathrm{ClO}_{4}\right]$ & $0.483 \pm 0.001$ & [14] \\
\hline
\end{tabular}

${ }^{\mathrm{a}}\left[\mathrm{BArF} \mathrm{B}_{24}\right]=\left[\mathrm{B}\left(\mathrm{C}_{6} \mathrm{H}_{3}\left(\mathrm{CF}_{3}\right)_{2}\right)_{4}\right]^{-;} ;{ }^{\mathrm{b}}[\mathrm{TFAB}]=\left[\mathrm{B}\left(\mathrm{C}_{6} \mathrm{~F}_{5}\right)_{4}\right]^{-;}{ }^{\mathrm{c}}$ the toluene: $\left[\mathrm{Bu}_{4} \mathrm{~N}\right]\left[\mathrm{BF}_{4}\right]$ electrolyte is of the $3: 1$ stoichiometry.

Table 1: Redox potentials of ferrocene in different organic media and supporting electrolytes. 
The solvent-Fc interaction is also present in ionic liquids. This issue was addressed by studying the effect of ionic liquid structure on the mid-point potentials of FC and DmFc in eleven different ILs as well as in dichloromethane with added IL as the supporting electrolyte [22]. As evidenced in Table 2, a variation in the $E_{m}$ of $\mathrm{Fc}$ vs. $\mathrm{DmFc}^{0 /+}$ of about $0.100 \mathrm{~V}$ is observed when the IL was changed from $\left[\mathrm{C}_{4} \mathrm{mim}\right]\left[\mathrm{CF}_{3} \mathrm{SO}_{3}\right]$ to $\left[\mathrm{C}_{2} \mathrm{mim}\right][\mathrm{FAP}]$ under neat conditions (water content $<100$ ppm). Similarly, when DmFc and Fc were simultaneously present in dichloromethane with added IL as the supporting electrolyte-conditions under which $\mathrm{DmFc}$ is less sensitive to solvation effects-a variation in the $E_{m}$ of Fc vs. $\mathrm{DmFc}^{0 /+}$ of about $0.050 \mathrm{~V}$ was observed when the supporting electrolyte was changed from $0.1 \mathrm{M}$ $\left[\mathrm{Et}_{4} \mathrm{~N}\right]\left[\mathrm{BF}_{4}\right]$ to $0.1 \mathrm{M}\left[\mathrm{C}_{2} \mathrm{mim}\right][\mathrm{FAP}]$. A variable potential difference which increases from $0.014 \mathrm{~V}$ in the case of [ $\left.\mathrm{C}_{4} \mathrm{mPyr}\right][\mathrm{FAP}]$ to $0.082 \mathrm{~V}$ in the case of $\left[\mathrm{C}_{4} \mathrm{mim}\right]\left[\mathrm{CF}_{3} \mathrm{SO}_{3}\right]$ is noticed from the comparison of the $E_{m}$ of $\mathrm{Fc} v$ s. $\mathrm{DmFc}^{0 /+}$ between neat ILs and diluted conditions (dichloromethane with added IL as the supporting electrolyte) [22].

\begin{tabular}{|c|c|c|c|c|}
\hline \multirow{2}{*}{ IL } & \multicolumn{2}{|c|}{$E_{\mathrm{m}}$ VS. $\mathrm{DmFc} 0 /+(\mathrm{V})$} & \multirow{2}{*}{$\frac{E_{\mathrm{m}} \mathbf{V s} . \mathbf{F} \mathbf{c}^{\mathbf{0} /+}(\mathrm{V})}{\mathrm{Cc}^{+}}$} & \multirow{2}{*}{ Ref } \\
\hline & Fc & $\mathrm{Cc}^{+}$ & & \\
\hline$\left[\mathrm{C}_{4} \mathrm{mPyr}\right][\mathrm{FAP}]$ & $0.575^{a}$ & $-0.747 a$ & $-1.322^{a}$ & {$[1,22]$} \\
\hline$\left[\mathrm{C}_{2} \mathrm{mim}\right][\mathrm{FAP}]$ & $0.574^{a}$ & $-0.749^{a}$ & $-1.323^{a}$ & {$[1,22]$} \\
\hline$\left[\mathrm{C}_{2} \mathrm{mim}\right]\left[\mathrm{B}(\mathrm{CN})_{4}\right]$ & $0.526^{a}$ & $-0.799^{a}$ & $-1.325^{a}$ & {$[1,22]$} \\
\hline$\left[\mathrm{C}_{2} \mathrm{mim}\right]\left[\mathrm{N}(\mathrm{Tf})_{2}\right]$ & $0.520^{a}$ & $-0.820^{a}$ & $-1.330^{a}$ & {$[23,24]$} \\
\hline$\left[\mathrm{C}_{4} \mathrm{mim}\right]\left[\mathrm{N}(\mathrm{Tf})_{2}\right]$ & $0.514^{a}$ & $-0.813^{a}$ & $-1.327^{a}$ & {$[1,22]$} \\
\hline \multirow{2}{*}[\mathrm{C}_{3}\mathrm{mPyr}]{$\left[\mathrm{N}(\mathrm{Tf})_{2}\right]$} & - & - & $-1.325^{b}$ & {$[25]$} \\
\hline & - & - & $-1.327^{b}$ & {$[25]$} \\
\hline \multirow{3}{*}[\mathrm{C}_{4}\mathrm{mPyr}]{$\left[\mathrm{N}(\mathrm{Tf})_{2}\right]$} & $0.511^{a}$ & $-0.816^{a}$ & $-1.327 a$ & {$[1,22]$} \\
\hline & - & - & $-1.363^{b}$ & [25] \\
\hline & - & - & $-1.333^{d}$ & [26] \\
\hline$\left[\mathrm{S}_{2} 21\right]\left[\mathrm{N}(\mathrm{Tf})_{2}\right]$ & - & - & $-1.348^{b}$ & [25] \\
\hline$\left[\mathrm{S}_{2} 2_{2}\right]\left[\mathrm{N}(\mathrm{Tf})_{2}\right]$ & - & - & $-1.354^{b}$ & [25] \\
\hline$\left[\mathrm{C}_{3}\right.$ mpip $]\left[\mathrm{N}(\mathrm{Tf})_{2}\right]$ & - & - & $-1.362^{b}$ & [25] \\
\hline$\left[\mathrm{HmimSC}_{4}\right]\left[\mathrm{N}(\mathrm{Tf})_{2}\right]$ & $0.510^{a}$ & $-0.830^{a}$ & $-1.330^{a}$ & [23] \\
\hline$\left[\mathrm{C}_{2} \mathrm{mim}\right][\mathrm{FSI}]$ & $0.512^{a}$ & $-0.820^{a}$ & $-1.332^{a}$ & {$[1,22]$} \\
\hline$\left[\mathrm{C}_{3} \mathrm{mPyr}\right][\mathrm{FSI}]$ & $0.510^{a}$ & $-0.822^{a}$ & $-1.332^{a}$ & {$[1,22]$} \\
\hline \multirow{2}{*}[\mathrm{C}_{2}\mathrm{mim}]{$\left[\mathrm{N}(\mathrm{CN})_{2}\right]$} & - & - & $-1.332^{b}$ & [25] \\
\hline & - & - & $-1.355^{b}$ & {$[25]$} \\
\hline$\left[\mathrm{C}_{4} \mathrm{mPyr}\right]\left[\mathrm{N}(\mathrm{CN})_{2}\right]$ & $0.505^{a}$ & - & - & {$[1,22]$} \\
\hline$\left[\mathrm{C}_{4} \mathrm{mim}\right]\left[\mathrm{PF}_{6}\right]$ & $0.478^{a}$ & $-0.851^{a}$ & $-1.329^{a}$ & {$[1,22]$} \\
\hline$\left[\mathrm{C}_{2} \mathrm{mim}\right]\left[\mathrm{BF}_{4}\right]$ & - & - & $-1.336^{c}$ & [27] \\
\hline$\left[\mathrm{C}_{4} \mathrm{mim}\right]\left[\mathrm{BF}_{4}\right]$ & $0.478^{a}$ & $-0.848^{a}$ & $-1.326^{a}$ & {$[1,22]$} \\
\hline \multirow{2}{*}[\mathrm{C}_{4}\mathrm{mim}]{$\left[\mathrm{CF}_{3} \mathrm{SO}_{3}\right]$} & $0.474^{a}$ & $-0.854^{a}$ & $-1.328^{a}$ & {$[1,22]$} \\
\hline & - & - & $-1.362^{b}$ & [25] \\
\hline$\left[\mathrm{C}_{4} \mathrm{mPyr}\right]\left[\mathrm{CF}_{3} \mathrm{SO}_{3}\right]$ & - & - & $-1.347^{b}$ & [25] \\
\hline
\end{tabular}

aPotentials were obtained using a scan rate of $0.1 \mathrm{Vs}^{-1} . E_{m}$ values were obtained with an accuracy of $\pm 0.003 \mathrm{~V}$ (n=5). ${ }^{\mathrm{b}} \mathrm{No}$ experimental error was reported. ${ }^{c} E_{m}$ values were obtained with an accuracy of $\pm 0.002 \mathrm{~V}$. ${ }^{\mathrm{d}} E_{m}$ value was obtained with an accuracy of $\pm 0.001 \mathrm{~V}$.

Table 2: Redox potentials of transition-metal sandwich complexes obtained by cyclic voltammetry in different ionic liquids.

The cobaltocenium|cobaltocene, $\mathrm{Cc}^{+/ 0}$ (also known as $\left.\left[\mathrm{Co}^{\mathrm{III}}\left(\eta^{5}-\mathrm{C}_{5} \mathrm{H}_{5}\right)_{2}\right]^{+} /\left[\mathrm{Co}^{\mathrm{II}}\left(\eta^{5}-\mathrm{C}_{5} \mathrm{H}_{5}\right)_{2}\right]\right)$, redox couple was used in organic solvents as an alternative IRRS. It was suggested by Strehlok, et al. in 1960 as an IRRS for organic solvent systems [28]. Although it was not considered by IUPAC as an alternative to the $\mathrm{Fc}^{0 /+}$ couple, it shows negligible volatility and high stability and solubility in some organic solvents [29] and ILs $[12,23,30,31]$. If the cathodic potential window of the solvent is large enough, two chemically reversible, one- 


\section{Medicinal \& Analytical Chemistry International Journal}

electron reduction processes can be observed (Eqs. 2 and $3)$.

$$
\begin{aligned}
& {\left[\mathrm{Co}^{\mathrm{III}}\left(\eta^{5}-\mathrm{C}_{5} \mathrm{H}_{5}\right)_{2}\right]^{+}+\mathrm{e}^{-} \leftrightarrows\left[\mathrm{Co}^{\mathrm{II}}\left(\eta^{5}-\mathrm{C}_{5} \mathrm{H}_{5}\right)_{2}\right]} \\
& {\left[\mathrm{Co}^{\mathrm{II}}\left(\eta^{5}-\mathrm{C}_{5} \mathrm{H}_{5}\right)_{2}\right]+\mathrm{e}^{-} \leftrightarrows\left[\mathrm{Co}^{1}\left(\eta^{5}-\mathrm{C}_{5} \mathrm{H}_{5}\right)_{2}\right]^{-}}
\end{aligned}
$$

The midpoint potential for the $\mathrm{Cc}^{0 /-}$ couple (Eq 3) was found to be considerably solvent dependent and not always can be determined accurately at room temperature, mainly due to the overlap and interaction of this process with the electrochemical response of the organic solvent used [29]. Thereby, only the $\mathrm{Cc}^{+/ 0}$ redox couple (Eq 2) has been extensively used in organic solvents to provide a known and stable reference point. As an extrapolation of concept, it is also used in IL systems. Surprisingly, little is known about the solvation effect of either different organic solvent with added supporting electrolytes or IL structure on the $\mathrm{Cc}^{+/ 0}$ formal potential [2].

Table 2 reports the IL effect on the $\mathrm{Cc}^{+/ 0}$ process using both $\mathrm{Fc}^{0 /+}$ and $\mathrm{DmFc}^{0 /+}$ as the IRRS. In the first case, the $E_{m}$ of $\mathrm{Cc}^{+/ 0}$ is almost constant and could be represented by a value of $1.327 \pm 0.005 \mathrm{~V}$ vs. $\mathrm{Fc}^{0 /+}$ in 14 different ILs and using glassy carbon as the working electrode (exceptions are $\left[\mathrm{C}_{2} \mathrm{mim}\right]\left[\mathrm{BF}_{4}\right]$ and $\left[\mathrm{C}_{4} \mathrm{mPyr}\right]\left[\mathrm{CF}_{3} \mathrm{SO}_{3}\right]$, where a value of 1.336 and $1.347 \mathrm{~V}$ was reported, respectively). Meanwhile, when $\mathrm{DmFc}^{0 /+}$ is used as the IRRS, an increase of $0.107 \mathrm{~V}$ in the potential separation between $\mathrm{Cc}^{+/ 0}$ and $\mathrm{DmFC}^{0 /+}$ couples is observed upon changing the IL from [C $\left.\mathrm{C}_{4} \mathrm{mPyr}\right][\mathrm{FAP}]$ to $\left[\mathrm{C}_{4} \mathrm{mim}\right]\left[\mathrm{CF}_{3} \mathrm{SO}_{3}\right]$.

To further understand this observation, we need to compare the variation in the $E_{m}$ of $\mathrm{Cc}^{+/ 0} \mathrm{vs}$. $\mathrm{Fc}^{0 /+}$ in organic solvents with added supporting electrolyte. It is well known that $\mathrm{Fc}$ is highly sensitive to solvation effects under these conditions (see above). As possible to see in Table 3, the $E_{m}$ of $\mathrm{Cc}^{+/ 0}$ in 9 different solvent/supporting electrolyte combinations can be represented by an almost constant value of about $1.352 \pm 0.015 \mathrm{~V}$ vs. $\mathrm{Fc}^{0 /+}$. As saw in Table 1, the $E_{m}$ value of the $\mathrm{Fc}^{0 /+}$ redox process varies from $0.437 \pm 0.005 \mathrm{~V}$ in ethanol $/ 0.1 \mathrm{M}\left[\mathrm{Bu}_{4} \mathrm{~N}\right]\left[\mathrm{ClO}_{4}\right]$ to a value of $0.532 \pm 0.002 \mathrm{~V}$ in dichloromethane/0.1M $\left[\mathrm{Bu}_{4} \mathrm{~N}\right]\left[\mathrm{ClO}_{4}\right]$, which represents a difference of $0.095 \mathrm{~V}$. Meanwhile, the obtained $\mathrm{Cc}^{+} E_{m}$ values vs. $\mathrm{Fc}^{0 /+}$ in the same two organic solvent systems (with $0.1 \mathrm{M}$ $\left[\mathrm{Bu}_{4} \mathrm{~N}\right]\left[\mathrm{ClO}_{4}\right]$ as the supporting electrolyte) show a

\begin{tabular}{|c|c|c|c|}
\hline Solvent & Electrolyte & $\mathrm{Cc}^{+/ 0}$ vs. Fc ${ }^{0 /+}(\mathrm{V})$ & Ref \\
\hline \multirow{3}{*}{ Acetonitrile } & $0.1 \mathrm{M}\left[\mathrm{Et}_{4} \mathrm{~N}\right]\left[\mathrm{ClO}_{4}\right]$ & $-1.350 \pm 0.003$ & {$[29]$} \\
\hline & $0.1 \mathrm{M}\left[\mathrm{Bu}_{4} \mathrm{~N}\right]\left[\mathrm{BF}_{4}\right]$ & $-1.348 \pm 0.003$ & \multirow{2}{*}{ [29] } \\
\hline & $0.1 \mathrm{M}\left[\mathrm{Bu}_{4} \mathrm{~N}\right]\left[\mathrm{PF}_{6}\right]$ & $-1.337 \pm 0.005$ & \\
\hline \multirow{2}{*}{ Dichloromethane } & $0.1 \mathrm{M}\left[\mathrm{Et}_{4} \mathrm{~N}\right]\left[\mathrm{ClO}_{4}\right]$ & $-1.359 \pm 0.007$ & {$[29]$} \\
\hline & $0.1 \mathrm{M}\left[\mathrm{Bu}_{4} \mathrm{~N}\right]\left[\mathrm{BF}_{4}\right]$ & $-1.355 \pm 0.007$ & [29] \\
\hline \multirow{2}{*}{ ethanol } & $0.1 \mathrm{M}\left[\mathrm{Et}_{4} \mathrm{~N}\right]\left[\mathrm{ClO}_{4}\right]$ & $-1.351 \pm 0.004$ & [29] \\
\hline & $0.1 \mathrm{M}\left[\mathrm{Bu}_{4} \mathrm{~N}\right]\left[\mathrm{BF}_{4}\right]$ & $-1.356 \pm 0.004$ & {$[29]$} \\
\hline Water & $0.1 \mathrm{M} \mathrm{Li}\left[\mathrm{ClO}_{4}\right]$ & $-1.367 \pm 0.002$ & [29] \\
\hline Toluene & $0.4 \mathrm{M}\left[\mathrm{P}_{66614}\right][\mathrm{FAP}]$ & $-1.340 \pm 0.005$ & {$[32]$} \\
\hline
\end{tabular}
variation of just $0.008 \mathrm{~V}$ (Table 3 ).

$\left[\mathrm{P}_{6} 66_{14}\right][\mathrm{FAP}]=$ trihexyl (tetradecyl)phosphonium tris(pentafluoroethyl)trifluorophosphate.

Table 3: Redox potentials of cobaltocenium hexafluorophosphate in different organic media and supporting electrolytes.

The comparison of data reported in the previous tables is of significant value as they suggest that the midpoint potentials of both $\mathrm{Cc}^{+/ 0}$ and $\mathrm{Fc}^{0 /+}$ couples are dependent on the solvation properties of organic solvents (with added supporting electrolyte) as well as the solvation properties of ILs, particularly under the assumption that $\mathrm{DmFc}^{0 /+}$ redox couple is a less solvent dependent process [2,22]. Consequently, potentials reported versus $\mathrm{Cc}^{+/ 0}$ and $\mathrm{Fc}^{0 /+}$ might need to be corrected in case of comparison of experimental data obtained in different organic solvents or ILs.

Other alternatives, such as 1,1'-dimethylferrocene, 1,1diacetylferrocene, ferrocenecarboxaldehyde, etc., could be used to provide a stable redox potential in organic systems $[23,30,33]$. However, these IRRS have not been fully characterised for this role as yet.

\section{Limitations in the Application of IRRS in Ionic Liquids}

- Poor solubility of Fc and DmFc in some ionic liquids, mostly in those with high viscosity $[31,34,35]$.

- Moderate volatility of Fc [35-37].

- Reactivity of $\mathrm{FC}^{+}$with the IL components: generally observed when the anodic potential window of the IL is close to the formal redox potential of $\mathrm{Fc}^{0 /+}[23,31]$. 


\section{Medicinal \& Analytical Chemistry International Journal}

- The $E_{m}$ of $\mathrm{FC}^{0 /+}$ redox process differs from the $E^{\theta^{\prime}}$ : this effect is related to the inequality in the diffusion coefficients of $\mathrm{Fc}$ and $\mathrm{Fc}^{+}$in almost all known ILs $[11,35]$.

- Reactivity of decamethylferrocene in the presence of oxygen [34].

\section{Selection of an Internal Reference Redox Scales for Voltammetric Measurements}

As a conclusion, good practice for electrochemistry dictates that the appropriated selection of the IRRS to provide a known and stable reference point in voltammetry is very important both for organic and IL solvent systems. The objective is to choose the IRRS (either the OXIRRS or RED IRRS component of a reversible redox couple) so that the cyclic voltammetric response for the simultaneously present electroactive ANS can be observed independently of the IRRS response. This means that if the ANS to be studied is in the oxidized form, then the reduced form of the IRRS must be added to the system. Conversely, if the ANS is in the reduced form, then the oxidized form of the IRRS must be added to the system. However, it is possible to see a large number of published works, which do not follow this basic rule [10].

The redox potential of $\mathrm{Fc}$ and $\mathrm{Cc}^{+}$are sensitive to the solvation properties of the organic solvent or IL components. Consequently, the use of $\mathrm{Fc}^{+} / 0$ and $\mathrm{Cc}^{+/ 0}$ couples as IRRS is expected to be adequate only when a single solvent system is being studied. However, corrections may be required in conditions that require comparison between different solvent systems. Even though DmFc is not freely soluble in ILs, the existing experimental results suggest that the $\mathrm{DmFc}^{0 /+}$ redox process is a suitable IRRS for voltammetric studies both in organic solvents and in ionic liquids, as it is less dependent on the solvent and supporting electrolyte nature in comparison to what is observed for ferrocene and cobaltocenium.

\section{References}

1. Torriero AAJ, Sunarso J, Howlett PC (2012) Critical Evaluation of Reference Systems for Voltammetric Measurements in Ionic Liquids. Electrochim Acta 82(1): 60-68.

2. Torriero AAJ (2015) Electrochemistry in Ionic Liquids. Volume 1: Fundamentals, Springer, Switzerland.
3. Inzelt G, Lewenstam A, Scholz F (2013) Handbook of reference electrodes. Springer, Berlin.

4. Bard AJ, Faulkner LR (2001) Electrochemical methods: fundamentals and applications. $2^{\text {nd }}(E d n$.$) ,$ John Wiley \& Sons, Inc., New York, NY, USA.

5. Izutzu $\mathrm{K}$ (2002) Electrochemistry in nonaqueous solutions. Wiley-VCH, NY, USA.

6. Smith TJ, Stevenson KJ (2007) Reference electrodes. In: Zoski CG (Eds.), Handbook of electrochemistry. Elsevier, Amsterdam, pp: 73-110.

7. Torriero AAJ, Bond AM (2009) Critical Evaluation of Electrochemistry in Ionic Liquids. In: Hayashi $\mathrm{K}$ (Eds.), Electroanalytical Chemistry Research Trends. Nova Science Publishers, Inc., New York.

8. Torriero AAJ (2019) Understanding the Differences between a Quasi-Reference Electrode and a Reference Electrode. Med Anal Chem Int J 3(3): 000144.

9. Gritzner G, Kuta J (1984) Recommendations on reporting electrode potentials in nonaqueous solvents. Pure Appl Chem 56(4): 461-466.

10. Torriero AAJ, Feldberg S, Zhang J, Simonov A, Bond A (2013) On choosing a reference redox system for electrochemical measurements: a cautionary tale. J Solid State Electrochem 17(12): 3021-3026.

11. Rogers EI, Silvester DS, Poole DL, Aldous L, Hardacre C, et al. (2008) Voltammetric Characterization of the Ferrocene/Ferrocenium and Cobaltocenium/Cobaltoc ene Redox Couples in RTILs. J Phys Chem C 112(7): 2729-2735.

12. Sukardi SK, Zhang J, Burgar I, Horne MD, Hollenkamp $\mathrm{AF}$, et al. (2008) Prospects for a widely applicable reference potential scale in ionic liquids based on ideal reversible reduction of the cobaltocenium cation. Electrochem Commun 10: 250-254.

13. Lewandowski A, Waligora L, Galinski M (2009) Ferrocene as a Reference Redox Couple for Aprotic Ionic Liquids. Electroanalysis 21(20): 2221-2227.

14. Noviandri I, Brown KN, Fleming DS, Gulyas PT, Lay PA, et al. (1999) The Decamethylferrocenium/Decam ethylferrocene Redox Couple: A Superior Redox Standard to the Ferrocenium/Ferrocene Redox Couple for Studying Solvent Effects on the Thermodynamics of Electron Transfer. J Phys Chem B 103(32): 6713-6722. 


\section{Medicinal \& Analytical Chemistry International Journal}

15. Barriere F, Geiger WE (2006) Use of Weakly Coordinating Anions to Develop an Integrated Approach to the Tuning of Delta E1/2 Values by Medium Effects. J Am Chem Soc 128(12): 3980-3989.

16. Ruiz-Diaz JJJ, Torriero AAJ, Salinas E, Marchevsky EJ, Sanz MI, et al. (2006) Enzymatic rotating biosensor for cysteine and glutathione determination in a FIA system. Talanta 68(4): 1343-1352.

17. Aranzaes JR, Daniel M-C, Astruc D (2006) Metallocenes as references for the determination of redox potentials by cyclic voltammetryPermethylated iron and cobalt sandwich complexes, inhibition by polyamine dendrimers, and the role of hydroxy - containing ferrocenes. Can J Chem 84(2): 288-299.

18. Ruiz J, Astruc D (1998) Permethylated electronreservoir sandwich complexes as references for the determination of redox potentials. Suggestion of a new redox scale. Comptes Rendus de l'Academie des Sciences Series IIc Chimistry 1(1): 21-27.

19. Matsumoto M, Swaddle TW (2004) The Decamethyl ferrocene $(+/ 0)$ Electrode Reaction in Organic Solvents at Variable Pressure and Temperature. Inorg Chem 43(8): 2724-2735.

20. Freyberg DP, Robbins JL, Raymond KN, Smart JC (1979) Crystal and molecular structures of decamethylmanganocene and decamethylferrocene. Static Jahn-Teller distortion in a metallocene. J Am Chem Soc 101(4): 892-897.

21. Bennett MA, Bhargava SK, Bond AM, Burgar IM, Guo SX, et al. (2010) Synthesis, X-ray structure and electrochemical oxidation of palladium(II) complexes of ferrocenyldiphenylphosphine. Dalton Trans 39(38): 9079-9090.

22. Torriero AAJ, Howlett PC (2012) Ionic liquid effects on the redox potential of ferrocene. Electrochem Commun 16(1): 84-87.

23. Torriero AA, Siriwardana AI, Bond AM, Burgar IM, Dunlop NF, et al. (2009) Physical and Electrochemical Properties of Thioether-Functionalized Ionic Liquids. J Phys Chem B 113(32): 11222-11231.

24. Huber B, Roling B (2011) Development of a Ag/Ag+ micro-reference electrode for electrochemical measurements in ionic liquids. Electrochim Acta 56(19): 6569-6572.
25. Lewandowski A, Waligora L, Galinski M (2013) Electrochemical Behavior of Cobaltocene in Ionic Liquids. J Solution Chem 42(2): 251-262.

26. Snook GA, Best AS, Pandolfo AG, Hollenkamp AF (2006) Evaluation of an Ag|Ag+ reference electrode for use in room temperature ionic liquids. Electrochem Commun 8(9): 1405-1411.

27. Waligora L, Lewandowski A, Gritzner G (2009) Electrochemical studies of four organometallic redox couples as possible reference redox systems in 1ethyl-3-methylimidazolium tetrafluoroborate. Electrochim Acta 54(5): 1414-1419.

28. Koepp ZM, Wendt H, Strehlow H (1960) Der Vergleich der Spannungsreihen in verschiedenen Solventien. II. Z Elektrochemie 64: 483.

29. Stojanovic RS, Bond AM (1993) Examination of conditions under which the reduction of the cobaltocenium cation can be used as a standard voltammetric reference process in organic and aqueous solvents. Anal Chem 65(1): 56-64.

30. Hultgren VM, Mariotti AWA, Bond AM, Wedd AG (2002) Reference Potential Calibration and Voltammetry at Macrodisk Electrodes of Metallocene Derivatives in the Ionic Liquid [bmim][PF6]. Anal Chem 74(13): 3151-3156.

31. Zhao C, Burrell G, Torriero AAJ, Separovic F, Dunlop NF, et al. (2008) Electrochemistry of Room Temperature Protic Ionic Liquids. J Phys Chem B 112(23): 6923-6936.

32. Duffy NW, Bond AM (2006) Macroelectrode voltammetry in toluene using a phosphoniumphosphate ionic liquid as the supporting electrolyte. Electrochem Commun 8(5): 892-898.

33. Bhatt AI, Bond AM, MacFarlane DR, Zhang J, Scott JL (2006) A critical assessment of electrochemistry in a distillable room temperature ionic liquid, DIMCARB. Green Chem 8(2): 161-171.

34. Torriero AAJ, Sunarso J, Forsyth M, Pozo-Gonzalo C (2013) Assessment of permethylated transition-metal sandwich complexes as internal reference redox systems in ionic liquids. PCCP 15(7): 2547-2553.

35. Torriero AAJ (2014) Characterization of decamethylferrocene and ferrocene in ionic liquids: argon and vacuum effect on their electrochemical properties. Electrochim Acta 137: 235-244. 


\section{Medicinal \& Analytical Chemistry International Journal}

36. Fu C, Aldous L, Dickinson EJ, Manan NS, Compton RG (2011) The kinetics of ferrocene volatilisation from an ionic liquid. Chemphyschem 12(9): 1708-1713.
37. Fu C, Aldous L, Dickinson EJF, Manan NSA, Compton RG (2011) Volatilisation of ferrocene from ionic liquids: kinetics and mechanism. Chem Commun 47(25): 7083-7085. 